\title{
Artificial Rearing of Mouse Pups: Development of a Mouse Pup in a Cup Model
}

\author{
ELIZABETH A. BEIERLE, MIKE K. CHEN, JOSEPH E. HARTWICH, MEERA IYENGAR, WEI DAI, \\ NAN LI, VINCE DEMARCO, AND JOSEF NEU
}

Department of Surgery [E.A.B., M.K.C., M.I., W.D.], Division of Pediatric Surgery, University of Florida, Gainesville, FL 32610, U.S.A.; University of Florida School of Medicine [J.E.H.], Gainesville, FL 32610, U.S.A.; Department of Pediatrics [N.L., J.N.], Division of Neonatology, University of Florida, Gainesville, FL 32610, U.S.A.; and Department of Child Health [V.D.], Division of Cardiology, University of Missouri, Columbia, MO 65211, U.S.A.

\begin{abstract}
Artificial rearing of rat pups has been used in the investigation of the neonatal gut. We propose to adapt the model of artificially rearing rat pups for use in mouse pups, thereby allowing the use of transgenic animals for our research. We hypothesized that gastrostomy catheters may be placed successfully into neonatal mouse pups and that the pups may be artificially reared without significant alterations in their growth or intestinal development. Gastrostomy tubes are placed into 5-d-old mouse pups [artificially reared (AR); $n=32]$, and the mice are fed rodent milk substitute. Littermate pups [maternally reared (MR); $\mathrm{n}=22$ ] are used as controls. After $5 \mathrm{~d}$, pups are killed and their organs are harvested. Intestinal villus measurements, protein content, and DNA content are determined. Data are reported as mean \pm SEM, compared with appropriate statistical methods, and significance is determined at $P<0.05$. Initial weights and lengths are not
\end{abstract}

\section{ABSTRACT}

different between the two groups, but after $5 \mathrm{~d}$, MR pups weigh more than their AR counterparts $(5.0 \pm 0.13$ versus $4.1 \pm 0.14 \mathrm{~g}$, MR versus AR; $P<0.01$ ). However, the pups' length and the intestinal villus height-to-width ratios, protein, and DNA content are not different between the MR and AR pups. To our knowledge, this is the first report of artificially rearing mouse pups. Development of this technique will permit nutritional manipulation in neonatal mice, a mammalian model wherein the genome is sequenced and transgenic mutants are available. (Pediatr Res 56: 250-255, 2004)
$\mathbf{A R}$, artificially reared
MR, maternally reared
RMS, rodent milk substitute

The nutritional status of the newborn and its effects on disease states and intestinal development have long been an area of intense study. Animal model systems are widely used and have proved to be effective for the investigation of a number of illnesses, with the findings extrapolated to the human condition. The development of a rodent in vivo animal model to study neonatal nutrition has been difficult, primarily because during the time that the animal is suckling from the mother and when the intestine is developing rapidly, it is difficult to control the amount and composition of the diet of the experimental neonate.

The technique of implanting an intragastric cannula for artificially rearing rat pups is well established (1). This technique allows for the study of a number of neonatal conditions

Received August 6, 2003; accepted March 34, 2004.

Correspondence: Elizabeth A. Beierle, M.D., Department of Surgery, P.O. Box 100286 JHMHSC, University of Florida, Gainesville, FL 32610; e-mail: beierea@surgery.ufl.edu This work supported by Children's Miracle Network Grants 2001-054 and 2002-011. N.L., V.D., and J.N. were partially supported by grant NIH HD R0138954.

DOI: 10.1203/01.PDR.0000132753.81333.39 with consistent access to the gastrointestinal tract, allowing for the control and manipulation of both the quantity and the composition of dietary intake in the preweanling rat pups. This model has been used extensively to study a myriad of conditions, including the determination of the nutritional demands for normal growth and development (2). It has been used in studies of intestinal maturation and enzymatic development (3). It has also been used to evaluate the effects of different insults, growth factors, and nutrients on the newborn gut $(4,5)$. The rat pup in a cup model has even been used to study the effects of nutrition (6) and toxin ingestion (7) on early brain development. Although the utility of the rat pup in a cup model has been extensive, it is not useful for studies in the transgenic animals that are currently available.

Currently, the primary laboratory animal used in transgenic research is the mouse. Numerous strains of genetically manipulated mice have been developed and are available for research purposes. If the rat pup in a cup model could be modified and developed for use in mouse pups, then the utility of the model and the conditions that could be studied would be increased. 
Our purpose is to develop a technique whereby the rat pup in a cup model may be adapted for the infant mouse.

\section{METHODS}

The University Veterinarian and the Institutional Animal Care and Use Committee of the University of Florida approved the following study. Swiss Webster time-dated pregnant mice were obtained from Charles River Laboratories (Wilmington, MA, U.S.A.). When the pups reached $5 \mathrm{~d}$ of age, the litters were divided into two groups, artificially reared (AR; $n=63$ ) and maternally reared (MR; $n=22$ ) animals. Intragastric feeding tubes were placed into the AR animals. The methods used are an adaptation of those described by Hall in 1975 (1) (Fig. 1). The tubes were placed in the following manner. Pups
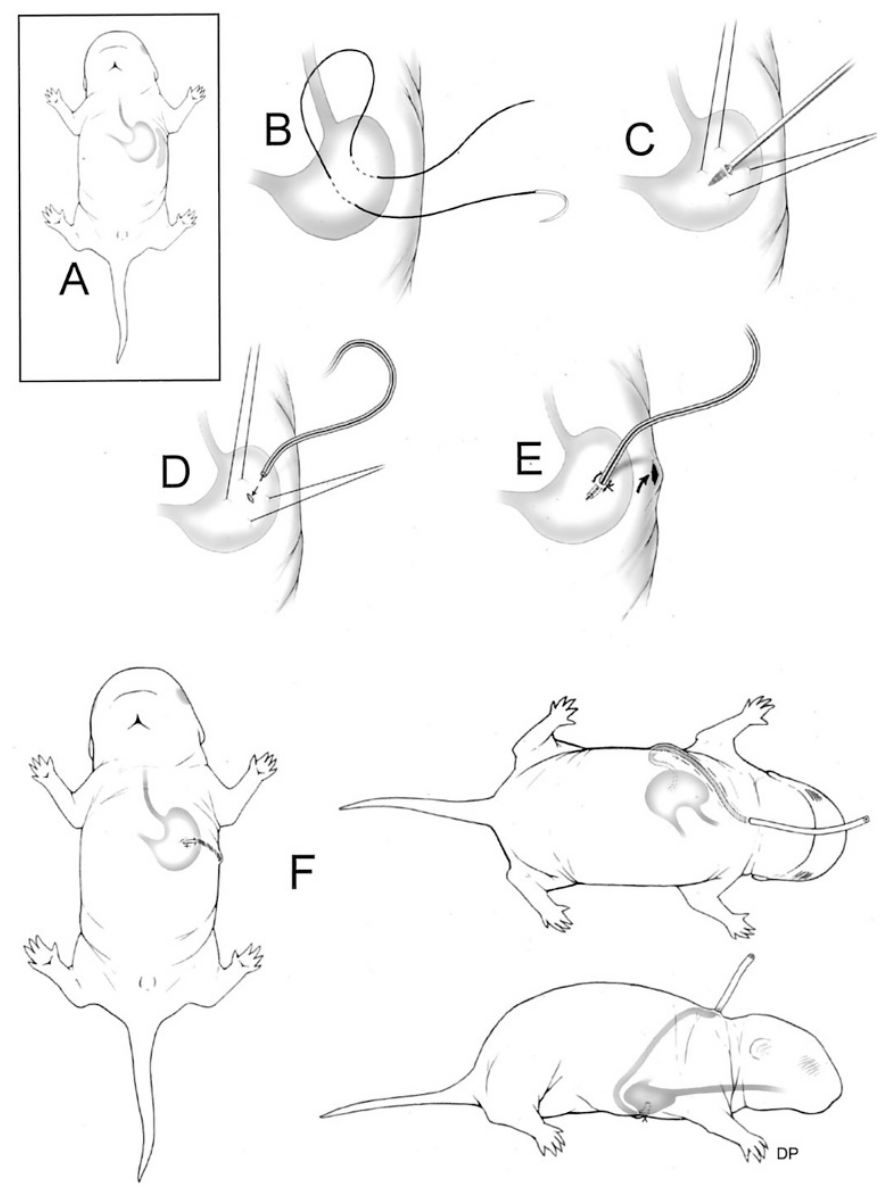

Figure 1. Method of gastrostomy insertion. This is an illustration of the percutaneous insertion of gastrostomy tubes into mouse pups. (A) The mouse pup is in the ventral view, with the stomach and spleen visible. The milk-filled stomach of the pup is easily seen through the transparent skin of the anterior abdominal wall. $(B)$ After adequate anesthesia, a small stitch in a "U" manner is placed through the abdominal wall incorporating the anterior wall of the stomach, thus pulling the stomach up tight to the abdominal wall. $(C)$ A needle is used to create a small stab wound through the skin and into the anterior gastric wall. $(D)$ A thin wire is passed through the stab incision into the stomach, and polyethylene tubing is passed over the wire into the stomach. $(E)$ The pursestring suture is tied, and the wire and tubing are tunneled under the skin of the abdomen toward the thorax. $(F)$ The wire and tube are tunneled under the skin of the thorax, out the nape of the neck, and the wire is removed. The final tunneled gastrostomy tube is depicted in the ventral, dorsal, and lateral views. were anesthetized with isoflurane inhalational anesthetic, and the stomach was visualized through the skin. The stomach is easily seen through the skin because the skin of the pup is fairly translucent and the stomach is white in color from the mother's milk. A figure-of-eight pursestring suture was placed through the skin and into the anterior wall of the stomach, pulling the stomach up tight against the anterior abdominal wall. A 20gauge needle creates a small stab wound through the skin and into the anterior wall of the stomach. An 8-gauge wire was placed through the skin opening, between the loops of the pursestring suture, into the stomach, and a short piece of \#10 polyethylene tubing was passed over the wire into the stomach. The wire was removed and the pursestring suture was tied to hold the tubing in place. The tubing was then tunneled under the skin to an exit site at the nape of the pup's neck (Fig. 1). The MR pups were given anesthesia, allowed to recover, and returned to their mothers for normal suckling.

Upon first attempting this procedure, we encountered some pitfalls. The first problem was splenic injury when placing the U-stitch. The spleen abuts the lateral wall of the stomach, and significant hemorrhage results when the spleen is damaged. In the mouse pup, the spleen is visible through the skin, and as long as the pup has recently eaten and has a full stomach, the spleen is easily avoided. The second most common problem that we faced was placing the polyethylene tubing too far into the stomach, resulting in the perforation of the back wall of the stomach. This problem was avoided by placing an ink mark on the tubing at the correct depth of insertion. The final problem was that of tying the suture too tight around the tubing, resulting in an obstruction of the gastrostomy tube. We solved this problem by paying particular attention to the tube when tying the suture and testing the patency of the tube with sterile water before completion of the procedure.

For feeding the AR pups, the intragastric cannula was connected to $\# 50$ polyethylene tubing that was connected to 3 -mL syringes filled with rodent milk substitute (RMS). The RMS used was mixed according to a formula previously published by Auestad et al. (2). The syringes with RMS are mounted on timer-controlled infusion pumps that are kept in a refrigerator to prevent the RMS from spoiling. Pups were fed $17 \mathrm{~min}$ each hour for $23 \mathrm{~h}$ per day. The volume of feedings was adjusted each day depending on the weight of the pups. A total volume of $\sim 0.3 \mathrm{~mL} \cdot \mathrm{g}^{-1} \cdot \mathrm{d}^{-1}$ was administered. This value is based on previous work in our laboratory with artificially rearing rat pups.

For regulating body temperature, the pups were kept in bedding-lined Styrofoam cups in a temperature-controlled water bath. MR pups and their lactating dams were housed in temperature-controlled rooms with light and dark controlled at 12-h on and off cycles.

The pups from both groups were weighed and measured daily. In addition, the AR pups were stimulated to urinate and defecate twice daily by gently massaging the genital area with moist, cotton tipped applicators. After $5 \mathrm{~d}$, pups from both groups were killed and their organs were harvested and snapfrozen in liquid nitrogen and then stored at $-80^{\circ} \mathrm{C}$ or placed into $10 \%$ buffered formalin for further studies. 
Formalin-fixed specimens of bowel were embedded in paraffin and cut. The sections of bowel were then stained with hematoxylin and eosin. Light microscopy images were captured through an Olympus $\mathrm{BH} 50 \mathrm{~A}$ microscope (Olympus America Inc., Melville, NY, U.S.A.) fitted with a JVC DC-150 digital camera (Micro Optics, Davie, FL, U.S.A.). Using a personal computer, villus height was measured in the proximal and distal small bowel. The epithelium of the villi was measured at three points, at the tip and on each side, to be certain that the villus had not been cut obliquely, and these measurements were used to obtain villus height. The villus height was calculated by first measuring from the tip of the villus to the base of the muscularis. Measurements were then taken from the base of the villus to the base of the muscularis on both sides of the villus, and these values were averaged. The averaged value from the bottom of the villus to the base of the muscularis was then subtracted from the first measurement, resulting in the true height of the villus. The width of the villus was measured, and the villus height-to-width ratios were calculated. Approximately 10 villi were measured per slide, and each pup had three stained slides, resulting in the analysis of $\sim 30$ villi per bowel segment.

Snap-frozen sections of intestine were used for total protein and DNA content determinations. Protein analysis was performed in the following manner. Specimens were weighed and lysed with $3 \mathrm{~mL} / \mathrm{g}$ of tissue lysis buffer containing $50 \mathrm{mM}$ of HEPES, $150 \mathrm{mM}$ of $\mathrm{NaCl}, 1 \%$ IGEPAL, $1 \mathrm{mM}$ of EDTA, and $1 \mathrm{mM}$ of $\mathrm{Na}_{3} \mathrm{VO}_{4}$. Specimens were then ground with mortar and pestle, boiled for $5 \mathrm{~min}$ at $100^{\circ} \mathrm{C}$, and centrifuged at $1200 \mathrm{rpm}$ at $-4^{\circ} \mathrm{C}$ for $15 \mathrm{~min}$. The suspension was collected and diluted 1:30 with water. Twenty microliters of each specimen was placed into a 96-well microplate. Dye reagent from Bio-Rad protein assay kit (1:5 dilution; Bio-Rad, Hercules, CA, U.S.A.) was added and, a plate reader was used to measure absorbance at 595 $\mathrm{nm}$. Results are reported as milligrams of protein per gram of tissue.

Quantification of DNA content of the intestine was performed using a DNeasy Tissue Kit (Qiagen, Valencia, CA, U.S.A.). The tissues were weighed and processed according to the package protocol, and DNA yield was determined by measuring the absorbance on a spectrophotometer at $260 \mathrm{~nm}$. Results are reported as micrograms of DNA per gram of intestinal tissue.

SigmaStat statistical analysis software (SPSS Inc., Chicago, IL, U.S.A.) was used for statistical calculations. Pup weight and length and protein and DNA data were evaluated with $t$ test. Villus height and villus height-to-width ratios were analyzed using an ANOVA. All data are reported as mean \pm SEM with $P \leq 0.05$ considered statistically significant.

\section{RESULTS}

We started with 63 pups in the AR group. Initial survival after surgery for the AR pups was $\sim 85 \%$ (53 of 63 ). During the period of artificial feeding, eight gastrostomy tubes malfunctioned before the end of the study period, and these pups were excluded from final analysis (three tubes were dislodged when sutures untied, and five tubes became irreversibly clogged). Thirteen (28\%) pups died during the study period. After $5 \mathrm{~d}$ of artificial rearing, 32 pups that had survived surgery were alive with intact, patent gastrostomy tubes. All (22 of 22) of the MR pups survived their anesthetic.

The weight $(3.51 \pm 0.08$ versus $3.38 \pm 0.13 \mathrm{~g}$, AR versus MR; $P=0.1)$ and the length $(6.5 \pm 0.08$ versus $6.7 \pm 0.12 \mathrm{~cm}$, AR versus $\mathrm{MR} ; P=0.2$ ) of the AR and the MR mouse pups were not significantly different at the initiation of the study (day of life 5). After $5 \mathrm{~d}$ (day of life 10), the MR pups weighed more than their AR counterparts $(5.0 \pm 0.13$ versus $4.1 \pm$ $0.14 \mathrm{~g}$, AR versus MR; $P<0.01$ ), but there were no differences in pup lengths between the two groups $(7.7 \pm 0.14$ versus $8.1 \pm 0.12 \mathrm{~cm}$, AR versus $\mathrm{MR} ; P=0.06$ ). Pup weight and length data are recorded in graphic form in growth curves in Figs. 2 and 3, respectively.

Hematoxylin and eosin staining of microscopic sections of the proximal and distal small bowel did not reveal any differences between the morphology of the bowel of AR versus MR mouse pups (Fig. 4). Villus measurements were determined from both the proximal and distal small bowel. Villus height in the proximal small intestine did not differ between AR and MR pups $(361.3 \pm 16.9$ versus $342.6 \pm$ $17.7 \mu \mathrm{m}$, AR versus MR; $P=0.4$; Fig. 5). The villus height-to-width ratio in this portion of the intestine also did not differ between AR and MR pups $(4.31 \pm 0.26$ versus $4.54 \pm 0.27$, AR versus MR; $P=0.7$; Fig. 6). Alternatively, the heights of the villi in the distal small intestine of AR pups were less than those of MR mouse pups $(252.8 \pm 11.9$ versus $288.7 \pm 10.2 \mu \mathrm{m}$, AR versus $\mathrm{MR} ; P=0.02$; Fig. 5). However, the height-to-width ratios of the villi in the distal small intestine were not significantly different between the two groups of pups $(3.46 \pm 0.11$ versus $3.55 \pm 0.11$, AR versus $\mathrm{MR} ; P=0.6$; Fig. 6 ).

\section{Growth Curve Weight (gm) Artificial vs Maternal Reared Mouse Pups}

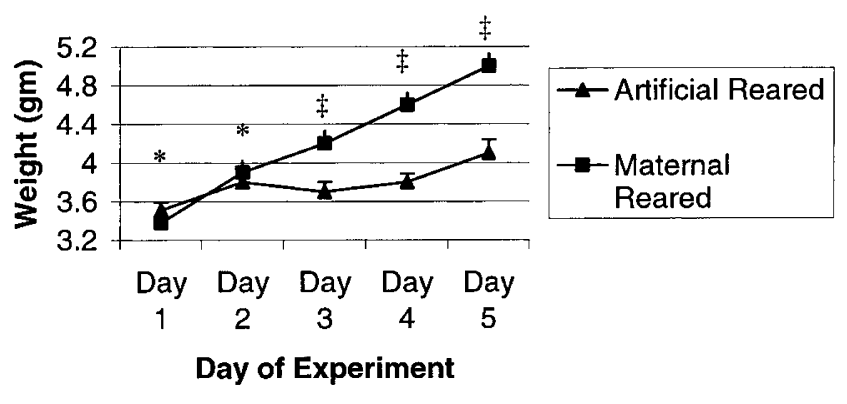

\section{* P $>0.05$ Artificial vs Maternal \\ $\ddagger \mathbf{P}<\mathbf{0 . 0 1}$ Artificial vs Maternal}

Figure 2. Growth curve weight (g), AR vs MR mouse pups. Initially, on day 1 of the study, there are no significant differences in the weights of the AR and MR pups $(3.51 \pm 0.08 v s 3.38 \pm 0.13 \mathrm{~g}$, AR $v s \mathrm{MR} ; P=0.4)$. However, after 5 experimental days, the AR pups weighed less than their MR counterparts (4.1 \pm 0.14 vs $5.0 \pm 0.13 \mathrm{~g}$, AR vs MR; $P<0.01)$. 
Growth Curve Length (cm) Artificial vs Maternal Reared Mouse Pups

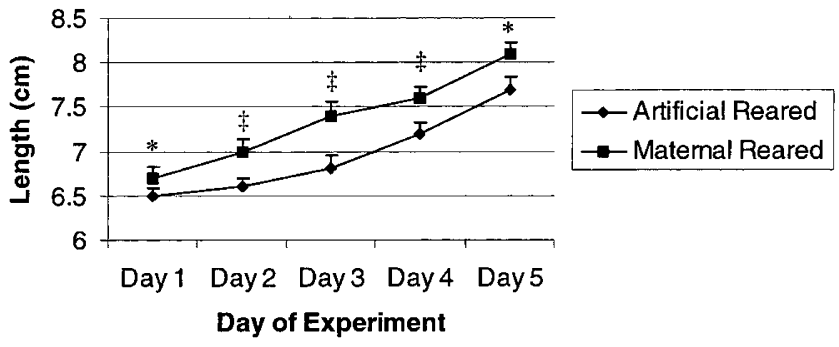

\section{* P > 0.05 Artificial vs Maternal Reared Pups \\ $\ddagger \mathbf{P}<\mathbf{0 . 0 5}$ Artificial vs Maternal Reared Pups}

Figure 3. Growth curve length (cm), AR vs MR mouse pups. Initially, on day 1 of the study, there were no differences in the length of the AR and the MR pups $(6.5 \pm 0.08 v s 6.7 \pm 0.12 \mathrm{~cm}, \mathrm{AR} v s \mathrm{MR} ; P=0.2)$. On days $2-4$ of the study, the length of the AR pups was below that of their MR counterparts. By day 5 of the study, the AR pups had caught up in their length and were not significantly different from the MR pups $(7.7 \pm 0.14 v s 8.1 \pm 0.12 \mathrm{~cm}$, AR $v s$ MR; $P=0.06)$.

\section{Proximal Small Intestine}

AR

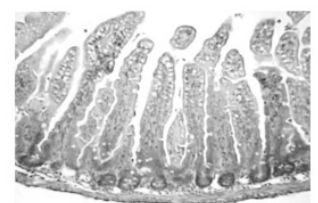

MR

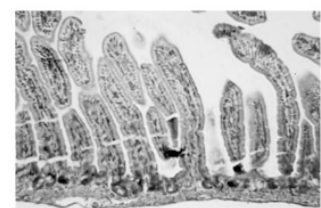

AR

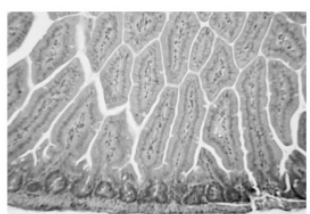

MR

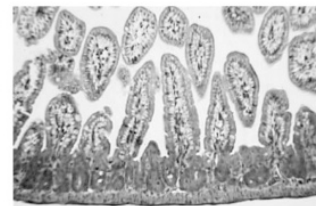

Figure 4. Micrographs of proximal and distal small intestine in MR vs AR mouse pups. Hematoxylin and eosin staining of sections of proximal and distal small intestine. There are no significant histologic differences in either the proximal or the distal small intestine between AR and MR mouse pups.

There were no differences in the amount of protein per gram of tissue $(6.42 \pm 0.3$ versus $7.2 \pm 0.2 \mathrm{mg} / \mathrm{g}$, AR versus $\mathrm{MR}$; $P=0.8)$ or in the amount of DNA per gram of tissue in the intestine of $\mathrm{AR}$ and MR mouse pups $(47.8 \pm 14.7$ versus 51.2 $\pm 20.5 \mu \mathrm{g} / \mathrm{g}$, AR versus MR; $P=0.9)$. These data are presented graphically in Figs. 7 and 8, respectively.

\section{DISCUSSION}

The insertion of feeding tubes into the gastrointestinal tract of rat pups for artificial rearing has been fully described. Differing methods have been used, ranging from surgical gastrostomy (8) to oral/percutaneous insertion of gastrostomy tubes (1). We believe that the utility of the rat pup in a cup model would be increased dramatically if it could be adapted for use in mouse pups, because the availability of transgenic

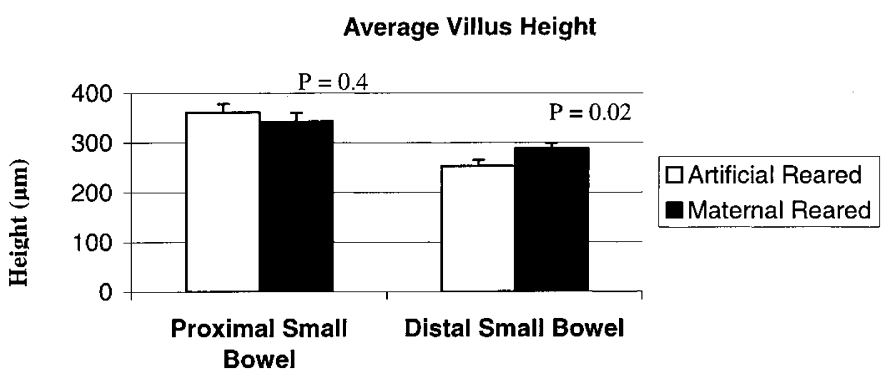

Figure 5. Average villus height. There is no significant difference in the average villus height between AR and MR pups in the proximal small bowel $(361.3 \pm 16.9$ vs $342.6 \pm 17.7 \mu \mathrm{m}$, AR vs MR; $P=0.4)$. However, in the distal small bowel, the AR animals tended to develop shorter villi than their MR controls $(252.8 \pm 11.9$ vs $288.7 \pm 10.2 \mu \mathrm{m}$, AR $v s \mathrm{MR} ; P=0.02)$.

\section{Villus Height to Width Ratio}

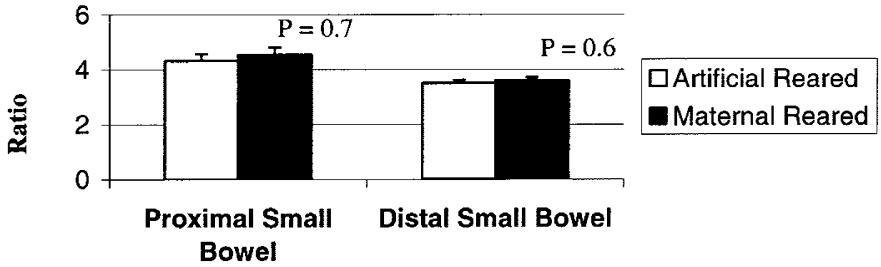

Figure 6. Villus height-to-width ratio. There is no significant difference in the villus height-to-width ratio in the proximal small bowel between AR and MR pups (4.31 $\pm 0.26 v s 4.54 \pm 0.27$, AR $v s \mathrm{MR} ; P=0.7)$. Despite differences in villus height in the distal small bowel between AR and MR pups, there is no significant difference in the height-to-width ratio in this area of the small intestine between the two groups $(3.46 \pm 0.11$ vs $3.55 \pm 0.11$, AR vs MR; $P$ $=0.6$ ).

\section{Intestine Protein Content}

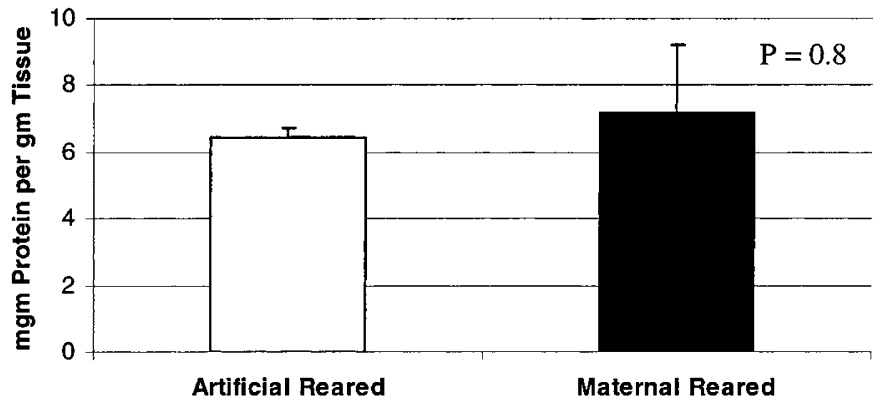

Figure 7. Intestine protein content. The amount of protein $(\mathrm{mg})$ per gram of intestinal tissue in AR vs MR mouse pups was determined. There is no significant difference in the amount of protein in the intestine between AR and MR pups $(6.42 \pm 0.3$ vs $7.2 \pm 2.0 \mathrm{mg} / \mathrm{g}$, AR vs MR; $P=0.8)$.

and knockouts is significantly greater for this species. The primary obstacles to using these techniques with mouse pups were the diminutive size of the pups and their increased sensitivity to anesthetic agents. Through the use of smaller instruments, less bulky materials, and minor alterations in both surgical and anesthetic techniques, we were able to successfully adapt our methods of gastrostomy tube insertion in rat pups for use in mouse pups.

The second objective of this study was to determine whether mouse pups could be artificially reared using the gastrostomy tubes and RMS. We were able to demonstrate comparable growth between mouse pups that were AR and 
Intestine DNA Content

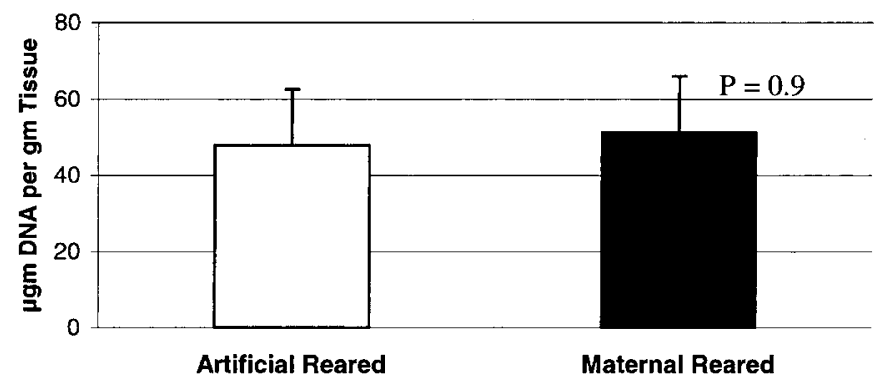

Figure 8. Intestine DNA content. The amount of DNA $(\mu \mathrm{g})$ per gram of intestinal tissue was determined in AR vs MR mouse pups. There is no significant difference in the DNA content in the intestine between AR and MR pups $(47.8 \pm 14.7$ vs $51.2 \pm 20.5 \mu \mathrm{g} / \mathrm{g}$, AR vs MR; $P=0.9)$.

those that were MR. The AR pups tended to show less weight gain than their MR counterparts but did not differ significantly in length at the end of the study period. Other authors have reported similar findings in rat pups reared with artificial feedings. Hall (1) found that the growth of rat pups that were reared artificially with RMS lagged behind the growth of MR pups. Smart et al. (9) showed smaller fat deposits in AR rat pups compared with those that were allowed normal suckling.

One explanation for the differences in pup weights in our study may be that during the first postoperative $24 \mathrm{~h}$, the AR pups were given only half-strength formula and were advanced to full-strength formula after the first $24 \mathrm{~h}$ postoperatively. This alteration in feeding was necessary as we found early in the study that the administration of full-strength RMS immediately after inhalational anesthesia and surgical intervention was too stressful for the pups' gastrointestinal tract and led to the early death of the pups. The animals developed abdominal bloating and gross changes in the intestinal tract that resembled findings noted in necrotizing enterocolitis, with distended bowel loops, pneumatosis intestinalis, and intestinal necrosis. In addition, we found that the small volume of the pups' stomachs restricted the amount of the feedings, thereby limiting the utility of increased volume as a method to improve weight gain. If given too large a volume, the pups would reflux the feeding or would become distended.

Evaluation of the length growth curve suggests that the AR pups do begin to exhibit catch-up growth after an initial lag of $\sim 1$ d. Perhaps decreasing the duration of half-strength feeds from 24 to 12 or $6 \mathrm{~h}$ may allow the pups' intestine adequate time to recover from the surgical and anesthetic stress, while placing the pups at less of a nutritional deficit at the initiation of the study. Finally, because this model is to be used to study the effects of feedings and gut development, we wished to determine whether artificial rearing would affect intestinal development. We found no gross morphologic differences by histologic examination between the intestines of MR or AR animals. The villus heights in the proximal small bowel were maintained in the AR animals but they were decreased in the distal small bowel of the AR group. These findings contrast those of Dvorak et al. (10), who has shown increased villus length and other histologic changes in the proximal small bowel of rat pups that are reared artificially on RMS compared with those that are MR or those that are AR with rat milk. Although the villus height in the distal bowel was less in the distal small bowel of the AR pups, the villus height-to-width ratio was unchanged in either the proximal or the distal small bowel of the two groups, suggesting that there were no changes in the actual absorptive surface area. We chose to use villus height-to-width ratios to compare the intestine between the two groups, as this is a method that has been reported in the literature by other laboratories as well as our own (11-13), and we believe that it is a better estimate of the true area available for absorption than either the villus height or width alone. Additional indices of intestinal development, including protein and DNA content of the intestine, were not affected by artificial rearing of the mouse pups.

Although we have demonstrated that mouse pups may be reared artificially without significant deleterious effects, some limitations to the methods remain. Despite the best attempts to mimic natural mouse milk with the RMS, there may be unknown factors present or absent from the milk substitute that may be responsible for changes in organ development, such as growth factors and hormones (4). In previous studies, investigators demonstrated that rat pups that were reared artificially with various substitutes showed differences in their organ development $(8,9,14)$. Most of these earlier studies, however, used RMS with a lower protein and higher carbohydrate concentration than is used in our current study. We used a milk substitute devised by Auestad et al. (2) that more closely mimics rat milk in its protein, carbohydrate, lipid, and caloric content. In Auestad's original report of this formulation, the only differences noted were an increase in intestinal length in AR rat pups, findings validated in later studies by Dvorak et al. (10).

To our knowledge, this is the first reported use of surgically implanted feeding tubes to artificially rear mouse pups. The mouse pups grow at a rate comparable to their MR counterparts with no significant alterations in intestinal development. Mice are the most widely used mammalian models in which the genome is sequenced and genetic mutants are readily available. Development of this technique will provide a means to perform studies involving nutritional manipulation in neonatal mice.

\section{REFERENCES}

1. Hall WG 1975 Weaning and growth of artificially reared rats. Science 190:13131315

2. Auestad H, Korsak RA, Bersgstrom JD, Edmond J 1989 Milk-substitutes comparable to rat's milk; their preparation, composition and impact on development and metabolism in the artificially reared rat. Br J Nutr 61:495-518

3. Yeh KY, Yeh M 1993 Use of pup in a cup model to study gastrointestinal development: interaction of nutrition and pituitary hormones. J Nutr 123:378-381

4. Staley MD, Gibson CA, Herbein JF, Grosvenor CE, Baumrucker CR 1998 Rat milk and dietary long arginine 3 insulin-like growth factor I promote intestinal growth of newborn rat pups. Pediatr Res 44:512-518

5. Yeh KY, Du FW, Holt PR 1986 Endogenous corticosterone rather than dietary sucrose as a modulator for intestinal sucrase activity in artificially reared rat pups. J Nutr 116:1334-1342

6. Diaz J, Samson HH 1980 Impaired brain growth in neonatal rats exposed to ethanol. Science 208:751-753

7. West JR 1993 Use of pup in a cup model to study brain development. J Nutr $123: 382-385$

8. Messer M, Thoman EB, Galofre A, Dallman T, Dallman PR 1969 Artificial feeding of infant rats by continuous gastric infusion. J Nutr 98:404-410 
9. Smart JL, Stephens DN, Katz HB 1983 Growth and development of rats artificially reared on a high or a low plane of nutrition. Br J Nutr 49:497-506

10. Dvorak B, McWilliam DL, Williams CS, Dominguez JA, Machen NW, McCuskey RS, Philipps AF 2000 Artificial formula induces precocious maturation of the small intestine of artificially reared suckling rats. J Pediatr Gastroenterol Nutr 31:162-169

11. Park YK, Dudley MA, Burrin DG, Donovan SM 2001 Intestinal protein and LPH synthesis in parenterally fed piglets receiving partial enteral nutrition and enteral insulinlike growth factor 1. J Pediatr Gastroenterol Nutr 33:189-195
12. Kisielinski K, Willis S, Prescher A, Klosterhalfen B, Schumpelick V 2002 A simple new method to calculate small intestine absorptive surface in the rat. Clin Exp Med 2:131-135

13. Potsic B, Holliday N, Lewis P, Samuelson D, DeMarco V, Neu J 2002 Glutamine supplementation and deprivation: effect on artificially reared rat small intestinal morphology. Pediatr Res 52:430-436

14. Diaz J, Moore E, Petracca F, Schacher J, Stamper C 1982 Artificial rearing of rat pups with a protein-enriched formula. J Nutr 112:841-847 